XXIV.

THE PRACTICAL USES OF RECENT WORK ON THE INTERNAL EAR, TO THE GENERAL PRACTITIONER, THE OTOLOGIST, OPHTHALMOLOGIST, SYPHILOLOGIST, NEUROLOGIST AND SURGEON.*

By IsaAC H. Jones, A. M., M. D.,

\title{
PHILADELPHIA.
}

Any work in physiology may be regarded as an "interesting study," but from the standpoint of most of us in the practice of medicine, research work of any kind becomes of greatest importance when it proves useful in clinical work. The cry is: "That is all very interesting, but of what use is it? How does it help sick people?" To answer this question is the spirit of this paper. A study of the relation of the ear to the central nervous system not only opens a new field for investigation in physiology, but also furnishes information of diagnostic value in the every-day examination of patients. Although it is essentially and entirely an ear study, and is useful in the analysis of ear conditions, we wish to emphasize that recent investigations have shown it to be of value in medical and surgical diagnosis.

It is now recognized that the internal ear, instead of being one organ, consists of two organs of different functionsthe cochlea, which is the organ of hearing, and the static labyrinth, which is the organ of equilibration. It seems very remarkable that whereas the cochlea has long been recognized as the organ of hearing, the static portion of the ear, consisting of the utricle, saccule and three semicircular canals, has been through all the years a "terra incognita." It is only in the past

* Read before the New York Academy of Medicine, Section on Otology, January 12, 1917, and published in the Journal of the American Medical Association. 
few years that the function of the static labyrinth has been carefully studied, preeminently by the Vienna group of otologists, to whom we are indebted for the new methods of testing the internal ear. Robert Bárány received the Nobel prize of 1915 for this work, and he is to be regarded as the pioneer in the clinical application of the study of the ear and the central nervous system.

\section{THE TESTS.}

The semicircular canals may be stimulated by revolving a person in a turning chair, or by douching the ears with either cold or hot water, or by applying the galvanic current to the ear. According to our experience, the turning and douching are much more useful than the electric test. The galvanic current stimulates equally all portions of the internal ear, and in fact the eighth nerve itself. As we regard it of greatest importance to analyze the function of the canals separately, we will consider in this paper only the tests by turning and douching. The essential feature in the physiology of these tests is that the endolymph within the particular canal or canals stimulated is set in motion on the one hand mechanically by the turning, and on the other hand thermically by the douching. When an individual is turned to the right, the endolymph catches up to the movement of the body, and when the turning chair is stopped, the endolymph continues to move to the right by its own momentum. The physiology of the caloric test may be illustrated by the "thermosyphon" system of some of the popular automobiles. They have no water pump to force a circulation through the radiator; the engine heats the water, decreasing its specific gravity, and causing it to move upward and forward into the radiator, where it is cooled, drops to the bottom of the radiator and continues downward and backward into the engine, thus completing the circuit. Similarly chilling the outer portion of a semicircular canal increases the density of the endolymph at this point and produces a circulation downward.

Such ear stimulation produces certain definite phenomena in normal individuals-a rhythmic jerking of the eyes, known as nystagmus, and a subjective sensation of turning, which may be termed a systematized vertigo. The eyes are always 
drawn in the direction of the endolymph movement, and are then quickly jerked back in the opposite direction by impulses from the cerebrum. The subjective sensation of vertigo is always in a direction opposite to the endolymph movement. According to our conviction, there are only two reactions produced by ear stimulation-vertigo and nystagmus; it is because of this vertigo that the individual falls in a definite direction, and also when he attempts to find with his finger an object he has previously touched, he is unable to find it, but "past-points" to the right or left, above or below, depending on the direction and plane of his sensation of vertigo.

It is evident that if stimulation of the ear causes a movement of the eyes, there must be a definite nerve pathway from the ear to the eye muscles. Also if ear stimulation produces dizziness, it is evident there must be a nerve pathway from the ear to the cerebral cortex. The special object of our work has been the attempt to determine the pathways through the brain from the ear. On the basis of over three hundred and fifty pathologic cases, including a considerable number of operations and autopsies, we have come to certain conclusions as to the course of the fibers from the ear through the brain stem, cerebellum and cerebrum. In brief, our conclusions are as follows: The fibers from the horizontal semicircular canals pass through the eighth nerve to the brain stem, and enter Deiters' nucleus in the medulla oblongata. At this nucleus the fibers divide, going on the one hand to the posterior longitudinal bundle, through which they are connected with the various eye muscle nuclei, to be distributed through the third, fourth and sixth nerves to the eye muscles themselves. It is this pathway that is responsible for the eye movement. The other pathway goes from Deiters' nucleus through the inferior cerebellar peduncle to the cerebellar nuclei of the same side, from which it proceeds through the right superior cerebellar peduncle to the decussation of the two superior cerebellar peduncles in the base of the cerebral crura; from this point there are two pathways to the cerebral cortex of both sides, but the main pathway goes to the cortex of the opposite side. The cortical centers that receive these fibers are postulated by Mills to be in the posterior portion of the second temporal convolutions adjacent to the cortical areas for hearing. It is 
this pathway from the horizontal canal to the cerebral cortex, passing through the cerebellum, that is responsible for the production of vertigo on ear stimulation. The fibers from the vertical semicircular canals have a different pathway after entering the brain stem, ascending in the pons to a point above the middle of the pons. At this point the fibers divide, going on the one hand to the posterior longitudinal bundle, to be distributed to the eye muscles; on the other hand the fibers enter the cerebellar nuclei through the middle cerebellar peduncle, from which point their pathway is the same as that of the fibers from the horizontal canal. In a nutshell, by the vestibular apparatus we mean the static portions of the internal ears and the pathways from the ears through the brain stem, cerebellum and cerebrum.

IMPORTANCE OF THESE TESTS IN DIAGNOSIS.

1. The general practitioner is constantly confronted with cases of "vertigo." It is sturely not overstating the fact to say that at best he can only guess as to its significance. In the light of these new tests, vertigo should no longer be regarded as a vague and general symptom. We should never speak of gastric vertigo, kidney vertigo, cardiovascular vertigo, idiopathic vertigo, or any other generalization which in its ultimate analysis means nothing. Vertigo is definitely a disturbance of the equilibratory apparatus. These tests give us the means of analyzing this apparatus. All conscious sensations are cerebral; headache, from whatever cause, is a disturbance in the brain. Similarly, vertigo is due to an irritation, impairment or destruction of some portion or portions of the ear or its associated pathways. Therefore, just as we examine the urine in suspected cases of nephritis or diabetes, or as we have a Wassermann test made in suspected cases of syphilis, just so these ear tests enable us to analyze the apparatus responsible for dizziness. Instead of guessing, we then know the reason for the vertigo. We do not hesitate to say that it is very rare that a "dizzy" case remains obscure after these ear tests, and in most instances the diagnosis becomes clear and simple. After these tests are made we are able to say, for example, that the patient is dizzy because of toxic impairment of his labyrinth, a labyrinthitis, an organic lesion in some 
part of the vestibular tract such as is produced, for example, by a brain tumor, internal hydrocephalus or multiple sclerosis; or in the presence of normal reactions, we can state with assurance that the vertigo is purely functional.

2. Value to the otologist. It is very surprising how much information can be obtained by these tests in the ordinary everyday study of an ear case. Briefly, from the ear standpoint alone, we have by means of the turning and douching tests, produced nystagmus, vertigo, past-pointing and falling, the clean-cut quantitative objective evidence of the function of each part of the static portion of the labyrinth and eighth nerve. A patient complains of deafness; the question arises, is it a middle ear or an internal ear condition? These hard and fast reactions give positive data of the function of the internal ear; then a positive opinion can be given with less reservation. Deaf and dumb institutions obviously should have a turning chair; in these poor unfortunates the functional tests of hearing are so often unsatisfactory; we never feel after making the hearing tests that we are sure the labyrinth is completely destroyed. It is just here that the new tests are valuable; they either do or do not give reactions. The answer is "Yes" or "No." In routine examination, it is, of course, not necessary to conduct all the tests; merely by turning the patient and obtaining twenty-six seconds of after-turning nystagmus, we at once can conclude that both static labyrinths are normal. Very often, however, the nystagmus will be subnormal, and immediately we have a hint as to the necessity for further study. We have no hesitancy in suggesting that if the otologist in his routine work will make such a simple test, he will be surprised to find that in many instances an entirely new light is thrown upon the condition of his patient.

3. Value to the ophthalmologist. The attention of the eye specialist has not been called to the intimate relation between the ear and the eye. He should surely know that a nystagmus of any type and in any direction can be produced "to order" by ear stimulation. There is an absolute and definite relation between the canal stimulated and the type of nystagmus produced. If we wish to produce a vertical nystagmus upward, we place the patient's head over toward the right shoulder, turn him to the left, and there appears a vertical nystagmus up- 
ward. If we wish a rotary nystagmus to the left, we douche the right ear with head upright with cold water, or douche the left ear with hot water, and there is produced a rotary nystagmus to the left. Although space does not permit discussing the reasons for this marvelously accurate ear-to-eye mechanism, yet the above gives some idea of the fixed relationship between the semicircular canals and the eye muscles. The "fistula" test serves as an illustration; if there is caries of bone in the outer wall of the labyrinth producing a fistula into the internal ear, or, for that matter, if the stapes is unduly mobile in the oval window, applying a Politzer bag into the ear and causing pressure or suction, causes the eyes to dance violently. Recently a well known ophthalmologist seeing us do this test expressed his astonishment-"I had no idea there was such close relation between the ear and the eye."

THE PRACTICAL APPLICATION OF THESE TESTS.

(a) In the study of eye muscle palsy. For example, loss of conjugate deviation in various directions should always be studied by means of the ear tests. There is a loss of voluntary control; the patient is unable, when asked, to look in a given direction. If the ear stimulus causes the eyes to move in this direction, it shows without question that there are normal pathways from the ear through the eye muscle nuclei and through the nerves to the eye muscles themselves-therefore, the lesion is supranuclear.

(b) Spontaneous nystagmus may be ocular in origin from corneal scars, macular lesions, or eye strain as in miners, in which case the cause is easily determined by proper ocular examination. But what of those cases in which the lesion lies not so near the surface? It is in such cases that the ear tests are essential. Recently there were two babies, both showing a wild oscillatory nystagmus. Naturally it was the ophthalmologist who was called on to determine its cause; he found nothing wrong in the eyes themselves to account for the nystagmus. The ear tests, however, showed a striking difference between the two cases. On one we reported: "The ear tests suggest an irritation of the vestibuloocular tract, but no organic impairment; the prognosis is good." The child had a perfect recovery. The report on the other patient was: 
"There is an organic block of the vestibuloocular tracts of both sides in the brain stem; the prognosis is bad." This patient died. The reason for this report was that the first patient on ear stimulation immediately showed a change of the oscillatory nystagmus into a definite rhythmic pull. The ear stimulation, as it were, seized both eyes and caused them to move rhythmically in the proper direction; evidently, therefore, there was no organic block of the pathways. The other case continued to have the oscillatory nystagmus and was entirely unaffected by ear stimulation, showing a definite organic block of the pathways from both ears to the eyes.

4. The ear and the syphilologist. Syphilis is such a prevalent affection that any method of diagnosis deserves thorough investigation. The disease manifests itself in so many forms that although it is comparatively easy to diagnose in certain cases, it is extremely difficult in others. There is probably no other disease where early diagnosis is so important; if detected early, certain well known remedies can often effect a prompt and permanent cure. Prognosis, in fact, depends largely upon how quickly the treatment is instituted. Discovery of the Wassermann test was justly hailed as an extraordinary aid to the clinician, but unfortunately the Wassermann reaction is not absolute; it is common experience to find cases that are clinically undoubtedly syphilitic, and which also improve under antisyphilitic treatment, but which are repeatedly negative to the Wassermann test. Our investigations to date have led us to believe:

(a) That the new ear tests may prove useful in the early diagnosis of syphilis. Many cases referred to us show a beginning very slight but unmistakable impairment of eighth nerve function only a few weeks after the primary infection; had it not been for the delicacy of the new tests, we would in all probability have not been able to detect this impairment for months.

(b) Ear examinations unquestionably are valuable in detecting a beginning involvement of the central nervous system. It is recognized that the nervous system is attacked in five per cent of those infected; we venture to suggest that probably this percentage is much higher, and that many cases are not recognized because there is no obvious involvement, as in locomotor ataxia, facial paralysis or general paresis. The eighth 
nerve is particularly vulnerable to this disease, and analysis of the function of the eighth nerve and the intracranial pathways from the eighth nerve may in certain cases demonstrate a beginning involvement of the nervous system several years before it can be detected by any other method.

5. Value to the neurologist. Naturally all the subjects already mentioned are of interest to the neurologist; the determining of the cause of vertigo, the degree of function of the eighth nerve and labyrinth, the study of eye muscle palsies, the analysis of spontaneous nystagmus, and the early diagnosis of syphilis of the central nervous system. In addition, the neurologist has in these tests a very definite help in:

(a) Differential diagnosis between labyrinth and intracranial lesions. The ear tests are indispensable for such differentiation. In so many instances the symptoms of internal ear disturbances'and of cerebellar lesions are identical. To illustrate: A patient was given a neurologic diagnosis of lesion of the right cerebellar hemisphere; ear tests gave additional data to the neurologist and demonstrated conclusively that it was a lesion of the right labyrinth.

(b) Intracranial localization. The accuracy of the ear tests has been very surprising in locating lesions in the cerebellar pontine angle, medulla oblongata, pons, cerebellar peduncles, cerebellum and various portions of the cerebrum, including the parietal lobe, temporal lobe and occipital lobe. Neurologists invariably want an eye examination in intracranial cases. We as otologists are not in a position to state the relative values, but the neurologists and ophthalmologists most acquainted with these ear tests assert that of the two methods of approach, very much more definite information can be had from the ear tests than from the eye examination.

6. Value to the surgeon. Perhaps no phase of surgery is so difficult in diagnosis as intracranial localization. It is a sad commentary on intracranial surgery that in a large majority of cases the surgeon must content himself merely with a palliative decompression. The ear tests have proven of real value in the locating of lesions; but in addition they may prove helpful to the surgeon, in preventing unnecessary operations. This may be illustrated by the following two cases: A woman appeared to have a tumor of the right cerebellar hemisphere. 
This diagnosis was confirmed by neurologic consultants, and the $\mathrm{X}$-ray report stated that it was a cyst in the right cerebellar hemisphere. The surgeon was about to operate, but hesitated because the ear report said it was a lesion of the right labyrinth, and that the cerebellum appeared intact. The woman had a perfect recovery without operation, has had a baby, and has remained in perfect health ever since-two years. Another woman was operated on for carcinoma of the gall bladder; the findings were negative. Subsequent ear examination made it perfectly simple that all her symptoms-nausea, vomiting, diarrhea and consequent emaciation-had been due entirely to recurring attacks of labyrinthitis. She made an excellent recovery. In the opinion of the surgeons most acquainted. with this work, no brain should be opened without giving the patient the benefit of the ear tests.

In conclusion, it must be remembered that the work of the ear specialist along the lines of the relation of the ear to medical and surgical diagnosis is only in its infancy. This paper is intended not so much to call attention to what we have done, as to hint at the future possibilities of this study and to indicate how the cooperation of the otologists with those in other branches of medical work can "help sick people." 